\title{
Kerr Beam Self-Cleaning in Multimode Fibers
}

\author{
A. Tonello ${ }^{1}$, R. Dupiol ${ }^{1,2}$, E. Deliancourt ${ }^{1}$, K. Krupa ${ }^{3}$, M. Fabert ${ }^{1}$, R. Guenard ${ }^{1}$, J. L. Auguste ${ }^{1}$, \\ A. Desfarges-Berthelemot ${ }^{1}$, V. Kermene ${ }^{1}$, A. Barthélémy ${ }^{1}$ D. Modotto $^{3}$, G. Millot ${ }^{2}$, S. Wabnitz ${ }^{3,4}$, V. Couderc ${ }^{1}$ \\ ${ }^{1}$ Université de Limoges, CNRS, XLIM, UMR 72521123 Avenue Albert Thomas, F-87000 Limoges, France \\ ${ }^{2}$ Université de Bourgogne Franche-Comté, ICB, UMR CNRS 6303, 9 Av. A. Savary, 21078 Dijon, France \\ ${ }^{3}$ Dipartimento di Ingegneria dell'Informazione, Università di Brescia, via Branze 38, 25123 Brescia, Italy \\ ${ }^{4}$ Novosibirsk State University, 1 Pirogova str., Novosibirsk 630090, Russia \\ Author e-mail address: alessandro.tonello@unilim.fr
}

\begin{abstract}
We overview recent experimental results of beam self-cleaning observed in various types of multimode fibers. We analyze the output spatial beam shapes and their connection with the refractive index profile of the fibers. (C) 2018 The Author(s)
\end{abstract}

OCIS codes: (190.4370) Nonlinear optics, fibers; (190.3270) Kerr effect; (190.4380) Nonlinear optics, four-wave mixing. (190.4420) Nonlinear optics, transverse effects.

\section{Introduction}

Several recent works in graded-index (GRIN) multimode fiber (MMF) have discussed the possibility of coupling, via the fiber nonlinearity, the spatial (one longitudinal, and two transverse) dimensions with the temporal dimension of pulsed beam propagation. Such novel phenomena have been initially observed in short segments of commercially available GRIN optical fibers. Among the several new phenomena observed in these fibers, the improvement of beam brightness at the output of GRIN MMFs [1,2] can stimulate further studies, including the development of new fiber designs, and the introduction of new materials. In what follows, we compare experimental observations of spatial beam self-cleaning in three different types of silica optical fibers.

\section{Experimental results}

Beam self-cleaning was observed for the first time in a commercially available GRIN-MMF using laser pulses of 900 ps at $1064 \mathrm{~nm}$ [1]. The panel (a) of Fig.1 summarizes the principal results: at the top, we show the measured refractive index profile of the GRIN fiber; middle and bottom frames show the wavelength and spatial content of beams at the output of a 3-m long fiber, after strong dispersion by a grating, at low and high power, respectively [1]. Those images show the output spectral densities as a function of wavelength and transverse coordinate $\mathrm{x}$ (at the position $\mathrm{y}=0$ ). It is evident that, for such long pulses, the Kerr effect improves beam brightness by squeezing the image in the horizontal dimension (spatial beam self-cleaning), while keeping a limited spectral broadening (as seen in vertical dimension).

As a further progress, it was recently shown that it is also possible to induce a quadratic nonlinearity through a process of optical poling in the same type of GRIN fiber [3,4]. For such an optically poled fiber, we observed the increase of the beam brightness not only for the pump but also for the generated second harmonic [3].

(a)
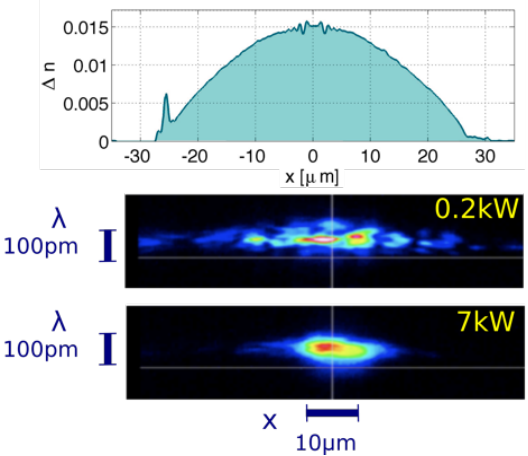

(b)
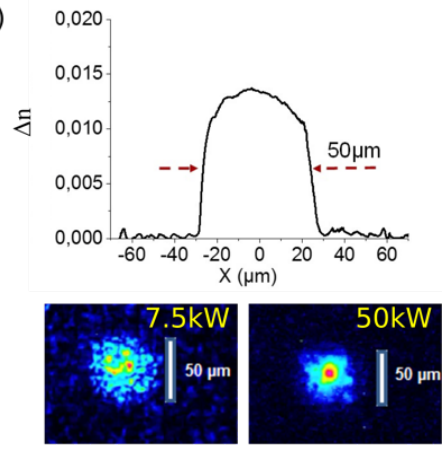

Fig. 1. (a) refractive index profile and spectrally dispersed output beam shapes at low power (speckled beam) and high power (cleaned beam) from a 3-m long GRIN fiber. (b) Refractive index profile and examples of output beam shapes at low power (speckled beam) and high power (cleaned beam) from a 3-m long Yb-doped fiber (passive configuration).

We also explored the cases of Yb-doped weakly guiding silica multimode fibers in passive and active configurations [5]. Panel (b) of Fig.1 summarizes the experimental results obtained with a Yb-doped MMF, where 
the measured refractive index profile is not of parabolic shape. In these experiments, we used a laser source emitting $500 \mathrm{ps}$ long pulses at $1064 \mathrm{~nm}$. The results are qualitatively similar to those observed in standard GRIN fibers (panel a), with a net improvement of beam brightness for laser powers above $40 \mathrm{~kW}$ (see lower frames of panel b).

We also investigated beam cleanup effects in an air-silica multimode microstructure fiber [6]. In Fig. 2 we show the results of a series of experiments carried out with 60 ps laser pulses at $1064 \mathrm{~nm}$. Panel (a) shows the hexagonal silica core surrounded by three layers of air holes, whose diameters gradually increase with the layer order. Panel (b) shows the evolution of output beam diameters for the pump (blue dots) and the $1^{\text {st }}$ Raman Stokes line (green dots) from a $11 \mathrm{~m}$ long fiber segment. When increasing the input pump power up to $20 \mathrm{~kW}$, Kerr self-cleaning leads to a limited spatial narrowing of the output beam diameter from $25 \mu \mathrm{m}$ down to $18 \mu \mathrm{m}$. In striking contrast with the cases shown in Fig.1, here the output pump beam diameter first reaches a minimum value, and then keeps growing larger for powers above $35 \mathrm{~kW}$. Such dynamics, which is different from that observed in GRIN fibers, may be explained by considering the relative high threshold power for Kerr beam cleaning, and the simultaneous competition of Raman scattering, which depletes the high central power region of the pump pulses. Raman conversion generates a Stokes beam which is particularly well localized at the center of the fiber core, owing to the modal dependence of the Raman gain for this fiber. Indeed, all Raman Stokes sidebands remain well localized, until they are depleted by the Raman conversion to next higher-order Stokes sideband. Note that in GRIN fibers and for longer pulses a similar effect of beam spreading at high powers has not been observed so far.

\section{(a)}

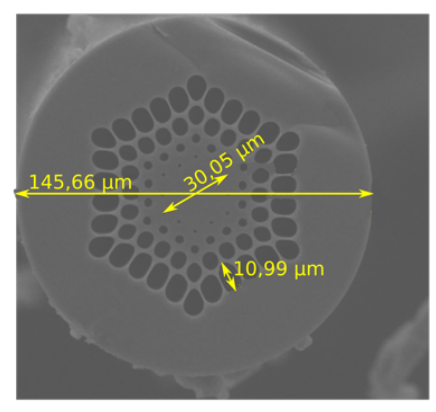

(b)

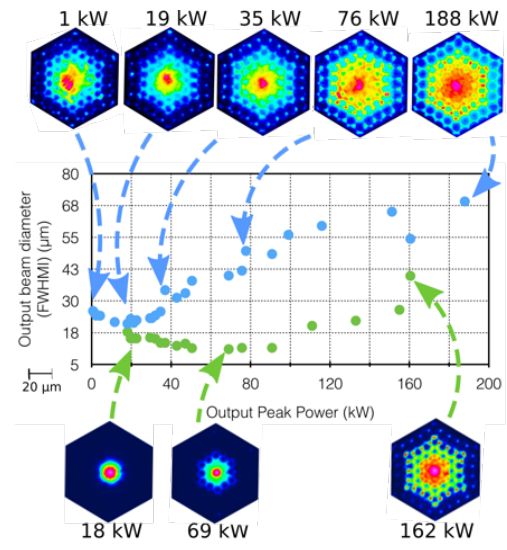

Fig. 2. (a) Scanning electron microscopy picture of the multimode microstructure fiber. (b) Evolution of the beam diameter for the pump (blue) and the $1^{\text {st }}$ Raman Stokes sideband (green) and corresponding beam shapes

In conclusion, we presented a series of experimental results showing a nonlinear modification of beam brightness at the output of different types of nonlinear MMF. Beam cleaning appears more effective in GRIN MMFs, but we observed a similar effect even in the case of an Yb-doped MMF. Kerr beam self-cleaning was also reported in a special type of microstructure MMF, but here the effect is eventually spoiled by Raman conversion and its corresponding Raman beam clean-up. All these phenomena were observed for the first time in microstructure fibers.

We acknowledge support from: the European Research Council (ERC) under the European Union's Horizon 2020 research and innovation programme, grant No. 740355; Marie-Sklodowska-Curie grant No. GA-2015-713694 (MULTIPLY); iXcore research foundation; Labex ACTION program (contract ANR-11-LABX-0001-01).

\section{References}

1. K. Krupa, A. Tonello, B. M. Shalaby, M. Fabert, A. Barthélémy, G. Millot, S. Wabnitz, and V. Couderc, "Spatial beam self-cleaning in multimode fibres," Nat. Photon. 11, 237-241 (2017).

2. Z. Liu, L.G. Wright, D.N. Christodoulides, F.W. Wise "Kerr self-cleaning of femtosecond-pulsed beams in graded-index multimode fiber," Opt. Lett. 41, 3675-3678 (2016).

3. D. Ceoldo, K. Krupa, A. Tonello, V. Couderc, D. Modotto, U. Minoni, G. Millot, and S. Wabnitz, "Second harmonic generation in multimode graded-index fibers: spatial beam cleaning and multiple harmonic sideband generation," Opt. Lett. 42, 971-974 (2017).

4. M. A. Eftekhar, Z. Sanjabi-Eznaveh, J. E. Antonio-Lopez, F. W. Wise, D. N. Christodoulides, and R. Amezcua-Correa, "Instant and efficient second-harmonic generation and downconversion in unprepared graded-index multimode fibers, ” Opt. Lett. 42, 3478-3481 (2017).

5. R. Guenard, K. Krupa, R. Dupiol, M. Fabert, A. Bendahmane, V. Kermene, A. Desfarges-Berthelemot, J. L. Auguste, A. Tonello, A. Barthélémy, G. Millot, S. Wabnitz, and V. Couderc, "Kerr self-cleaning of pulsed beam in an ytterbium doped multimode fiber," Opt. Express 25, 4783-4792 (2017).

6. R. Dupiol, K. Krupa, A. Tonello, M. Fabert, D. Modotto, S. Wabnitz, G. Millot, and V. Couderc, "Interplay of Kerr and Raman beam cleaning with a multimode microstructure fiber," Opt. Lett. 43, 587-590 (2018). 